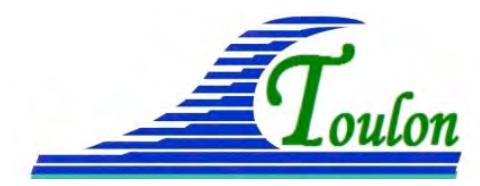

XIV èmes Journées Nationales Génie Côtier - Génie Civil

Toulon, 29 juin au $1^{\text {er }}$ juillet 2016

DOI:10.5150/jngcgc.2016.076 @ Editions Paralia CFL

disponible en ligne - http://www.paralia.fr - available online

\title{
Modélisation numérique à haute résolution de la submersion marine : plaine du Ceinturon, Hyères-les-Palmiers, Var
}

\author{
Sylvestre LE ROY ${ }^{1}$, Alexis STEPANIAN ${ }^{1}$, Rodrigo PEDREROS ${ }^{1}$, \\ Thomas BULTEAU ${ }^{1}$, Alexandre NICOLAE-LERMA ${ }^{1}$, Yann BALOUIN ${ }^{1}$
}

1. BRGM, Direction Risques et Prévention et Direction des Actions Territoriales, 3 avenue Claude Guillemin, BP 36009, 45060 Orléans Cedex 2, France

s.leroy@brgm.fr ; a.stepanian@brgm.fr ; r.pedreros@brgm.fr ; t.bulteau@brgm.fr ; a.nicolaelerma@brgm.fr;y.balouin@brgm.fr.

\section{Résumé :}

Cette étude, réalisée sur la ville d'Hyères-les-Palmiers (Var), vise à évaluer l'impact de l'élévation du niveau de la mer sous l'effet du changement climatique sur les submersions marines susceptibles d'affecter les zones urbanisées en contexte microtidal, notamment à proximité de petits estuaires. Les périodes de retour des événements (30, 50 et 100 ans) ont été déterminées par analyse multi-variée et calcul de probabilités conjointes des paramètres de forçages (vagues, niveau marin, niveau de la rivière). L'élévation du niveau de la mer (échéances 2030 et 2100) a été fixée conformément aux recommandations utilisées pour l'établissement des Plans de Prévention des Risques Littoraux (respectivement $+20 \mathrm{~cm}$ et $+60 \mathrm{~cm}$ ). Le modèle implémenté (SWAN pour la propagation spectrale des vagues, SWASH pour la submersion à terre par franchissement et/ou débordement) rend compte des processus hydrodynamiques à haute-résolution (de 1 à $5 \mathrm{~m}$ ), en intégrant notamment le bâti et l'occupation du sol. Après validation sur la submersion causée par la tempête de décembre 2008 (seul évènement récent de submersion suffisamment renseigné sur ce littoral), différents scénarios ont été simulés (périodes de retour 30 ans à échéance 2030 et 2100, et périodes de retour 50 et 100 ans à échéance 2100). Les résultats mettent en évidence la vulnérabilité des zones urbanisées littorales le long de la plaine du Ceinturon, et confirment certains éléments de connaissance sur les processus locaux, comme le rôle des lônes sur la propagation des eaux sur la zone arrière-littorale, les rôles relatifs des processus de franchissement et de débordement dans la submersion totale et le rôle des ouvrages. Ces résultats pourront désormais être utilisés par la municipalité pour orienter l'aménagement futur dans les zones exposées.

Mots-clés : Submersion marine, Tempête, Vague, Surcote, Franchissement par paquets de mer, Modélisation 2D, Zone urbaine.

\section{Introduction}

La simulation de la submersion marine par franchissement est un domaine en plein développement, du fait des récents progrès réalisés sur les modèles à résolution de 


\section{Thème 7 - Risques côtiers}

phase, sur les moyens informatiques et sur la disponibilité de données à haute résolution (topo-bathymétrie par méthodes LiDAR). Ces outils permettent désormais de passer des méthodes empiriques (généralement déterminées sur des cas idéalisés) à des simulations très fines capables de prédire de façon réaliste le comportement des écoulements face aux ouvrages de défense et à la présence de bâti (LE ROY et al., 2015).

La présente étude a été conduite pour le compte de la ville d'Hyères-les-Palmiers (Var), dans le cadre de l'appel à projet "Expérimentation de la relocalisation des activités et des biens : recomposition spatiale des territoires menacés par les risques littoraux", lancé en 2012 par le ministère en charge de l'environnement. L'objectif consistait, en s'appuyant sur ces nouveaux outils, à caractériser de façon réaliste la submersion marine sur le territoire de la plaine du Ceinturon (figure 1) et l'impact de l'élévation du niveau de la mer sur l'exposition de ce territoire à la submersion marine.
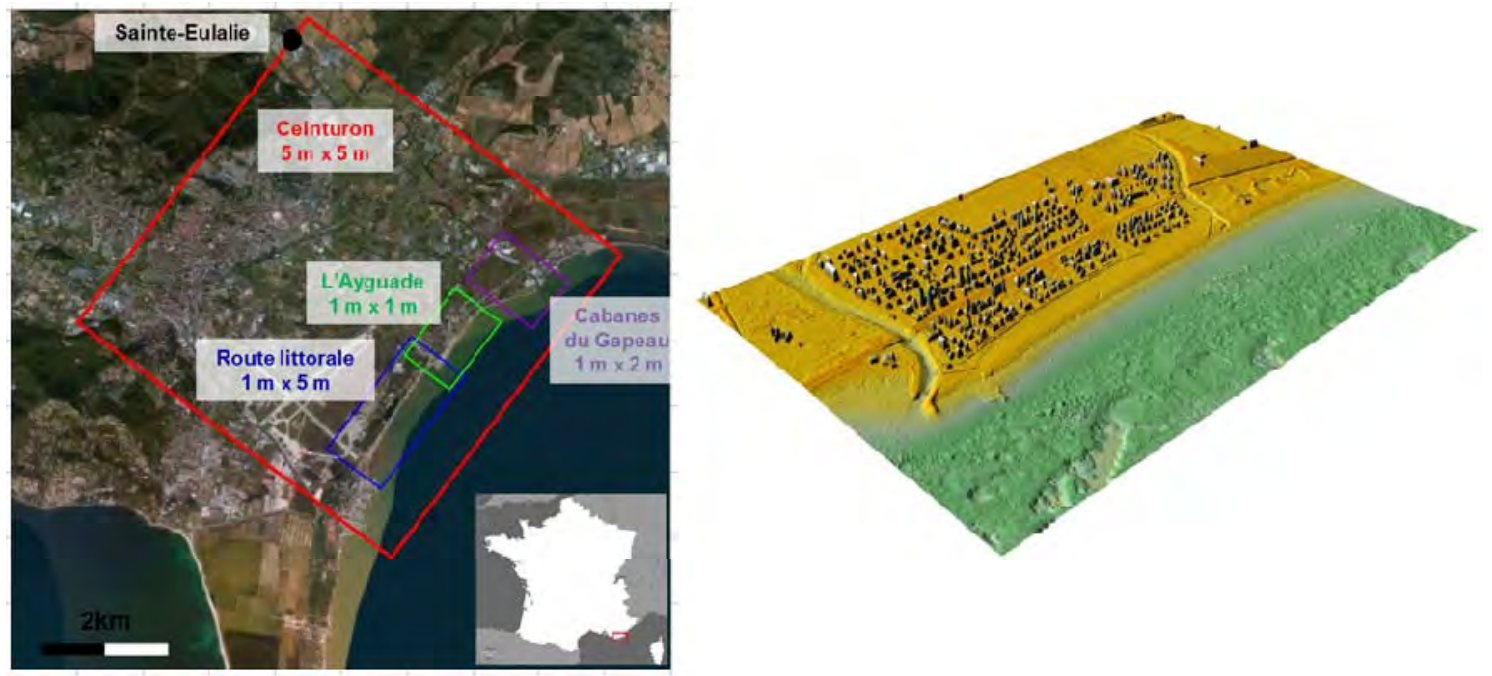

Figure 1. Aperçu de la zone d'étude et emprises des grilles de calcul utilisées (en haut) et modèle numérique d'élévation sur le hameau de l'Ayguade (en bas).

Pour cela, un modèle numérique permettant la simulation des processus de submersion a été mis en place, à haute résolution (prise en compte du bâti), en prenant en compte la diversité des phénomènes susceptibles d'interagir lors d'un évènement de tempête pour aboutir à une inondation des zone basses côtières (débordement, franchissement, concomitance entre surcote et crue de la rivière présente sur le site, le Gapeau).

En premier lieu, les scénarios de périodes de retour prédéfinies (30, 50 et 100 ans) ont été déterminés sur la base d'une analyse aux probabilités conjointes. Après validation de la chaine de modélisation, les simulations numériques de ces scénarios depuis le large jusqu'à la submersion ont alors permis d'estimer la submersion avec l'effet de l'élévation du niveau de la mer (échéances 2030 et 2100). Ces résultats ont alors permis d'analyser l'exposition future de ce territoire aux différents processus impliqués. 


\section{XIV èmes Journées Nationales Génie Côtier - Génie Civil \\ Toulon, 29 juin au $1^{\text {er }}$ juillet 2016}

\section{Détermination des scénarios}

\subsection{Données disponibles}

En l'absence de marégraphe sur le site, les mesures effectuées depuis 1961 (mais avec des lacunes significatives entre 1968 et 2012) au marégraphe de Toulon (à une vingtaine de kilomètres) ont été supposées représentatives du niveau marin à Hyères.

La caractérisation des vagues dans la rade de Hyères a quant à elle été effectuée à partir d'une base de données réalisée par ailleurs par le BRGM (STEPANIAN et al., 2014). Cette base, établie par des simulations spectrales des vagues sur la période 1979-2009 et validée sur des événements tempétueux, permet d'estimer les caractéristiques des vagues à une résolution d'ordre hectométrique dans ce secteur.

Enfin, le niveau de la rivière a été caractérisé à partir des mesures du limnigraphe de Sainte-Eulalie, situé environ 5 km en amont de l'embouchure. Les mesures exploitées, réalisées en continu, couvrent la période allant de fin 1970 à fin 2013.

\subsection{Méthode}

En raison d'un degré de dépendance non négligeable entre les variables, une analyse conjointe trivariée a été mise en œuvre à l'aide du logiciel Join-Sea (HR WALLINGFORD \& LANCASTER UNIVERSITY, 2000), portant sur les trois paramètres (niveau de la mer, HS des vagues au large, niveau de la rivière).

Les événements (triplets indépendants) ont été sélectionnés à partir des pics de crue et des maximums associés de niveau marin et de vagues (Hs et $\mathrm{Tp}$ associées), correspondants au final à 16,5 ans environ de données communes (73 évènements/an).

La distribution normale trivariée (dépendance entre les 3 variables) a été déterminée, ainsi que les distributions marginales (propres à chaque variable individuellement). Ces distributions ont été utilisées pour simuler par une méthode de Monte Carlo une période fictive de 100000 ans, (7 300000 triplets) permettant au final de calculer les surfaces de périodes de retour de dépassement conjoint (i.e. période de retour d'un événement pour lequel chacun des 3 paramètres va dépasser des valeurs données pour chacun). Pour en faciliter la lecture, ces surfaces ont ensuite été déclinées en courbes d’isoprobabilité de dépassement pour des niveaux donnés de la rivière (figure 2).

Enfin, sachant que les configurations les plus défavorables se trouvent dans la zone de courbure (niveau marin élevé et fortes vagues) et que l'objectif de l'étude est de se focaliser sur la submersion (niveau de la rivière peu élevé), un triplet a été sélectionné sur la courbe pour chaque période de retour étudiée afin d'élaborer les conditions de forçage au large pour les scénarios étudiés. Il convient toutefois de noter que les scenarii ainsi déterminés correspondent en fait à des niveaux d'eau totaux à la côte de périodes de retour plus faibles, puisque l'approche par probabilités conjointes conduit à considérer un dépassement conjoint des seuils à la fois en termes de niveau d'eau, de vagues et de niveau de la rivière. D’autre part, il n'a pas été possible dans le cadre de 


\section{Thème 7 - Risques côtiers}

cette étude d'explorer les différentes combinaisons de triplets de manière à identifier les configurations les plus défavorables en termes de submersion marine. La synthèse des paramètres retenus est présentée au tableau 1.

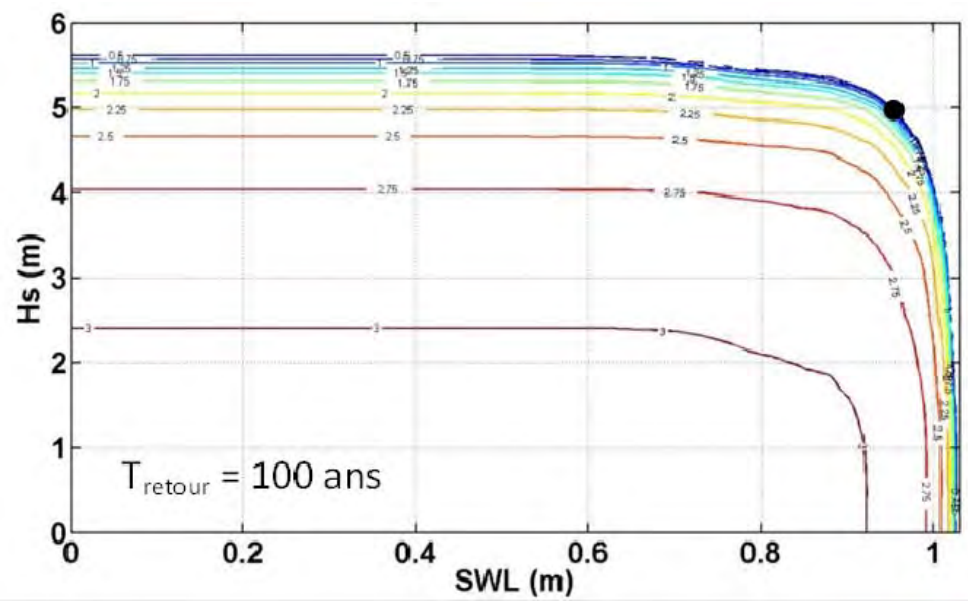

Figure 2. Contours centennaux de dépassement conjoint pour le niveau marin (SWL),la hauteur des vagues (Hs) et le niveau de la rivière ( $N R$, indiqué sur chaque courbe); le point noir représente le triplet centennal sélectionné.

Tableau 1. Synthèse des paramètres retenus pour l'élaboration des scénarios.

\begin{tabular}{|c|c|c|c|c|}
\hline Paramètre & $\begin{array}{l}\text { Tr=30 ans } \\
\text { Ech. } 2030\end{array}$ & $\begin{array}{l}\text { Tr }=30 \text { ans } \\
\text { Ech. } 2100\end{array}$ & $\begin{array}{l}\text { Tr }=50 \text { ans } \\
\text { Ech. } 2100\end{array}$ & $\begin{array}{l}\text { Tr=100 ans } \\
\text { Ech. } 2100\end{array}$ \\
\hline $\begin{array}{l}\text { Niveau de la mer actuel SWL } \\
\text { (m Cote marine) }\end{array}$ & 0.95 & 0.95 & 0.95 & 0.95 \\
\hline Vagues au large Hs (m) & 4.50 & 4.50 & 4.71 & 4.99 \\
\hline Niveau rivière $N R(m)$ & 0.50 & 0.50 & 0.50 & 0.50 \\
\hline $\begin{array}{l}\text { Elévation du niveau moyen de } \\
\text { la mer }(m)\end{array}$ & +0.20 & +0.60 & +0.60 & +0.60 \\
\hline $\begin{array}{l}\text { Niveau de la mer résultant (m } \\
N G F \text { ) }\end{array}$ & 0.90 & 1.30 & 1.30 & 1.30 \\
\hline
\end{tabular}

\subsection{Chronologie et résultats}

L'analyse des chronologies de quelques tempêtes récentes a montré que les pics des vagues et du niveau marin sont à peu près synchrones, alors que le pic de crue peut être quant à lui déphasé de plusieurs heures. Au final, il a donc été choisi de s’appuyer directement sur la chronologie de la seule tempête récente connue ayant occasionné une submersion significative (décembre 2008). L’évolution des paramètres lors de cette tempête a donc été normalisée sur une durée de 2,5 jours, puis imposée à chaque scénario à partir de leurs spécificités, l'élévation du niveau de la mer sous l'effet du changement climatique ayant été surimposée au niveau marin (les niveaux marins hors 


\section{XIV ${ }^{\text {èmes }}$ Journées Nationales Génie Côtier - Génie Civil \\ Toulon, 29 juin au $1^{\text {er }}$ juillet 2016}

tempête incluent cette élévation). Les séries temporelles des conditions de forçage (niveau marin, vagues au large, niveau de la rivière en amont) pour la chaine de modélisation ont ainsi été établies (exemples à la figure 3). On peut toutefois noter que compte-tenu des choix effectués, le forçage du niveau de la rivière reste constant.
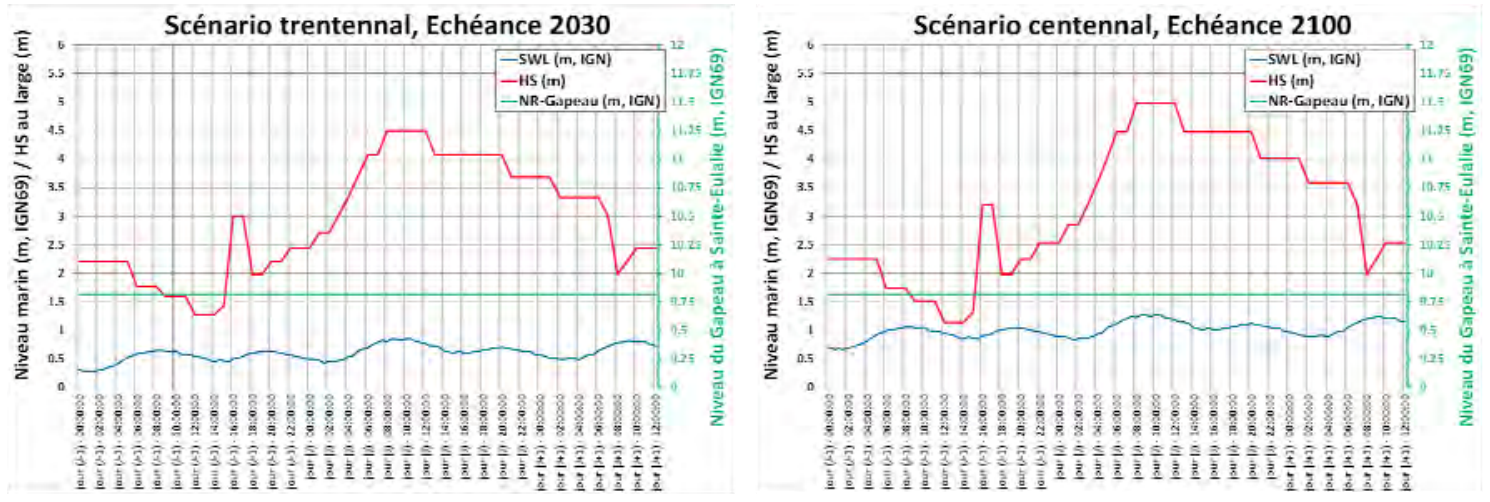

Figure 3. Aperçu des conditions de forçage pour les scénarios trentennal à échéance 2030 et centennal à échéance 2100.

\section{Simulations de la submersion}

La stratégie de simulation s'appuie sur une descente d'échelle et sur un choix des paramètres à considérer suivant la configuration. Elle a été validée sur la submersion occasionnée par la tempête de décembre 2008, puis appliquée aux scénarios réalisés.

\subsection{Propagation des vagues jusqu'à la côte}

Du fait de la transformation des vagues à l'approche de la côte, les caractéristiques des vagues (déterminées au large lors de l'analyse aux probabilités conjointes) ont été propagées jusqu'à la côte à l'aide d'un modèle spectral, avec une résolution suffisante pour percevoir les transformations qu'elles subissent à la côte. Le modèle spectral SWAN (BOOIJ et al., 2004), basé sur l'équation spectrale de conservation de l'action de la houle résolue suivant un schéma implicite en différences finies, a ainsi été implanté de manière à couvrir la rade d'Hyères à une résolution de $20 \mathrm{~m}$ (maillage régulier). La limite Est de l'emprise passe par le point pour lequel les vagues ont été caractérisées lors de l'analyse aux probabilités conjointes (Point P81, représenté à l'Est sur la figure 4). Les scénarios déterminés précédemment ont alors été simulés en mode instationnaire (niveaux d'eau et vagues) sur une période de 2,5 jours, moyennant quelques hypothèses (les vagues ont été supposées de secteur Est, conformément aux statistiques issues de la base de données de vagues en ce point, et elles sont supposées homogènes le long de la limite Est de l'emprise). 


\section{Thème 7 - Risques côtiers}

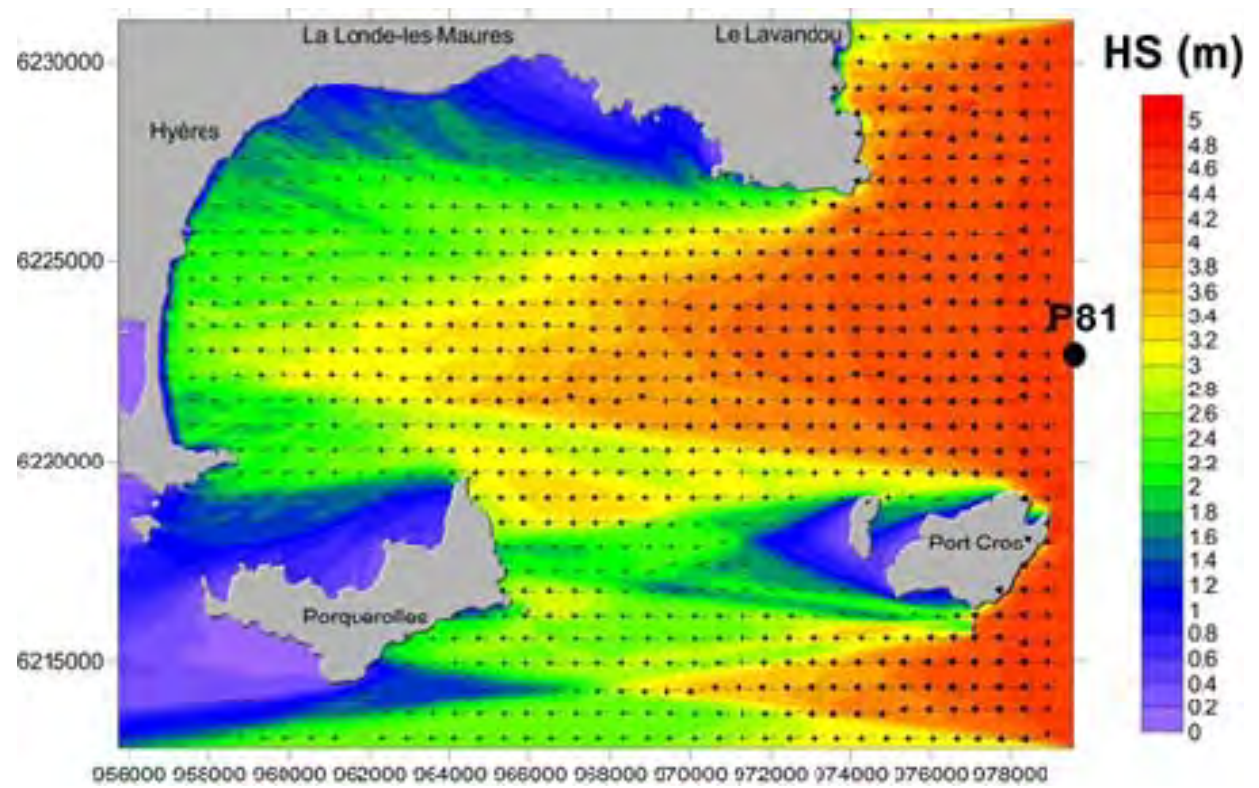

Figure 4. Emprise du domaine utilisé pour la propagation des vagues issues des scénarios jusqu'à la côte et aperçu des hauteurs significatives simulées au pic de la tempête (scénario trentennal, échéance 2030).

\subsection{Modèle}

SWASH est un modèle à résolution de phase ("vague-à-vague") développé par l'Université Technologique de Delft (ZIJLEMA et al., 2011). Il s'agit d'un modèle numérique non-hydrostatique, qui résout les équations de Saint Venant non linéaires (NLSW) en faible profondeur en y ajoutant un terme de pression non-hydrostatique (http://swash.sourceforge.net), permettant de simuler la propagation des vagues en domaine côtier et la submersion marine (réfraction, diffraction, frottement au fond, gonflement, déferlement, réflexion, interactions vague-vague et vague-courant, interface sec-mouillé, ...). Un aperçu d'une simulation SWASH est présenté à la figure 4, pour l'événement utilisé pour la validation de la chaine de modélisation (paragraphe 3.5).

Les simulations réalisées pour cette étude ont toutes été effectuées en coordonnées cartésiennes, en utilisant des coefficients de friction de Manning spatialisés et le modèle de Smagorinsky pour la viscosité turbulente. La prise en compte du déferlement des vagues a été activée, et la pression non-hydrostatique est intégrée avec un schéma numérique de type Keller-box. L'intégration temporelle est explicite avec des valeurs de CFL généralement comprises entre 0.3 et 0.5 , conduisant à des pas de temps dans les simulations typiquement de l'ordre de $0.02 \mathrm{~s}$. En ce qui concerne les conditions aux limites, les vagues sont forcées depuis le bord parallèle à la côte, alors que les autres limites marines reçoivent des éponges numériques de manière à amortir les éventuelles vagues qui peuvent s'en approcher. En raison d'instabilités numériques, les frontières terrestres sont toutefois représentées comme fermées (murs), ce qui conduit très 


\section{XIV èmes Journées Nationales Génie Côtier - Génie Civil \\ Toulon, 29 juin au $1^{\text {er }}$ juillet 2016}

probablement à une surestimation de l'inondation dans les zones en arrière pour les scénarios les plus impactants, puisque l'eau ne peut continuer à s’écouler vers l'arrière.

\subsection{Données d'entrée}

Afin de simuler les différents phénomènes sur la zone d'étude, plusieurs grilles de calcul ont été utilisées, d'emprises et de résolutions variables (figure 1).La plus grande emprise couvre l'ensemble de la plaine du Ceinturon jusqu'à Sainte-Eulalie afin de permettre de simuler l'écoulement de la rivière ; ce MNT (réalisé à partir des données LiDAR et des levers topo-bathymétriques disponibles) permet uniquement de simuler les phénomènes de débordement (marin et/ou fluvial) à une résolution de $5 \mathrm{~m}$, la composante dynamique des vagues n'étant pas prise en compte. L'ensemble du linéaire côtier de la plaine du Ceinturon a ensuite fait l'objet de simulation à des résolutions métriques, à partir de 3 grilles de calcul d'emprises plus restreintes, couvrant respectivement du Sud au Nord la route littorale, le hameau de l'Ayguade et les Cabanes du Gapeau. Ces grilles correspondent cette fois à des MNE (modèles numériques d'élévation, intégrant le bâti et certains murs dans la topographie, figure 1), réalisés essentiellement à partir des données LiDAR.

En complément, une cartographie de l'occupation du sol à l'échelle du territoire a été déclinée en grilles de friction à travers des coefficients de Manning représentatifs de la rugosité du sol. Les zones urbaines ont alors fait l'objet de traitements spécifiques puisqu'elles ont reçu des coefficients de Manning élevés $\left(0,1\right.$ à $\left.0,4 \mathrm{~s} / \mathrm{m}^{1 / 3}\right)$ sur la grille de $5 \mathrm{~m}$ de résolution (MNT sans bâtiments, d'où une forte rugosité), alors que de faibles valeurs des coefficients de Manning $\left(0,016 \mathrm{~s} / \mathrm{m}^{1 / 3}\right)$ leur ont été attribuées sur les grilles plus résolues (MNE intégrant le bâti, d'où une rugosité plutôt représentative du béton).

\subsection{Forçages}

Les conditions de forçage pour chacune des grilles de calcul sont déduites directement des scénarios déterminés lors de l'analyse probabiliste (§ 2.3) et de la simulation de la propagation des vagues à travers la rade d'Hyères (§ 3.1). Ainsi, la simulation à $5 \mathrm{~m}$ de résolution sur l'ensemble de la Plaine du Ceinturon est forcée d'une part par l'évolution du niveau du Gapeau au Nord et d'autre part par l'évolution du niveau de la mer (incluant le wave-setup calculé lors des simulations spectrales) côté marin. Les rangs de calculs plus fins sont quant à eux forcés par l'évolution du niveau marin et les caractéristiques des vagues côté marin, la grille des Cabanes du Gapeau (correspondant à l'estuaire de la rivière) recevant en plus le forçage du niveau de la rivière dérivé de la simulation à $5 \mathrm{~m}$ de résolution. De manière à faire varier les conditions de forçage dans le temps, les simulations de vagues ont été réalisées en conditions stationnaires (caractéristiques des vagues et du niveau de la mer) par période de 30 minutes, chaque période étant initialisée à partir des conditions finales de la période précédente. Les vagues sont par ailleurs générées à partir de spectres de type JONSWAP, caractérisés 


\section{Thème 7 - Risques côtiers}

par la hauteur significative des vagues, leurs périodes pic, leur directions pic et la dispersion directionnelle associée (tous ces paramètres étant issus des simulations spectrales en amont),

\subsection{Validation}

Les submersions marines ayant affecté le secteur d'étude restant rares, la stratégie de modélisation a été validée sur la tempête survenue le 14 décembre 2008, seul événement récent connu ayant causé une submersion par des franchissements de paquets de mer au niveau de l'Ayguade. La simulation de la submersion sur le rang de résolution $5 \mathrm{~m}$ s'avère compatible avec les observations (pas de débordement significatif, que ce soit côté fluvial ou côté marin, bien que la cote de la rivière à l'embouchure s'avère proche de celle des quais). La simulation de la submersion à très haute résolution au niveau de l'Ayguade s'avère également en accord avec les observations disponibles, quoique la submersion semble légèrement sous-estimée (ce qui peut en partie s'expliquer par la très faible inondation observée, avec des hauteurs d'eau du même ordre de grandeur que la précision estimée des données LiDAR) : l'eau ne pénètre la zone urbaine que par les vagues qui s'infiltrent au-delà du mur de haut de plage par les passages piétons aménagés (franchissement des vagues), conduisant à une inondation de quelques centimètres à quelques dizaines de centimètres dans les rues du front de mer (figure 5).

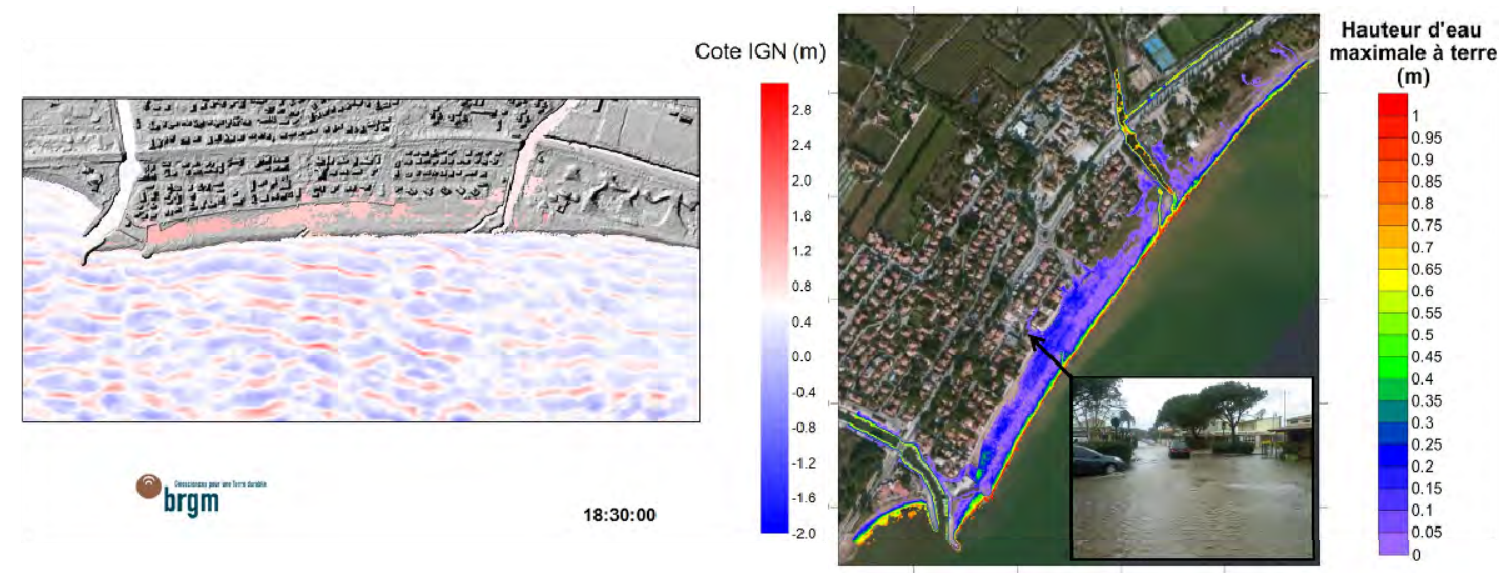

Figure 5. Aperçu instantané de la simulation SWASH sur l'Ayguade (en haut) et hauteurs d'eau maximales simulées à terre lors de la tempête du 14 décembre 2008 comparées à une photographie de l'inondation (photo : Mairie d'Hyères) (en bas).La

simulation couvre la période de $09 \mathrm{~h} 00$ à 18h00, avec en forçage des hauteurs significatives des vagues variant de 1.3 à $1.8 \mathrm{~m}$ pour des périodes de $6.8 \mathrm{~s}$ à $8.3 \mathrm{~s}$ et un niveau de la mer compris entre 0.38 et $0.62 \mathrm{~m} \mathrm{NGF}$. 


\section{XIV èmes Journées Nationales Génie Côtier - Génie Civil \\ Toulon, 29 juin au $1^{\text {er }}$ juillet 2016}

\section{Résultats}

Les simulations ont ainsi été menées sur chacune des grilles de calcul pour chaque scénario considéré. Le scénario centennal à échéance 2100 a été simulé en considérant une ruine généralisée des ouvrages de protection (ici le muret de haut de plage à l'Ayguade et l'enrochement aux Cabanes du Gapeau).

Les simulations à l'échelle de la Plaine du Ceinturon montrent que l'embouchure la rivière contribue au débordement de manière limitée (son débit restant très modéré). Les simulations à très haute résolution permettent quant à elles de mettre en évidence un certain nombre de conclusions sur le site d'étude :

a) Le site, bien qu'actuellement peu affecté par la submersion marine, pourrait être sensiblement impacté à l'avenir sous l'effet du changement climatique (notamment les hameaux de l'Ayguade et des Cabanes du Gapeau);

b) Les rôles relatifs des différents processus montrent que si les franchissements peuvent être significatifs à courte échéance même pour des événements relativement fréquents, le débordement devient à long terme le processus dominant sous l'effet de l'élévation du niveau de la mer, les vagues se révélant alors moins importantes ;

c) Les structures locales peuvent avoir un impact important sur la submersion, que ce soit en termes de protection (ouvrages de protection, sous l'hypothèse qu'ils résistent) ou en termes de faiblesse (défaillance des ouvrages, présence de "lônes" qui permettent un débordement dans les zones basses en arrière des zones urbanisées conduisant à une propagation plus importante de la submersion dans ces zones, voir par exemple la figure 6).
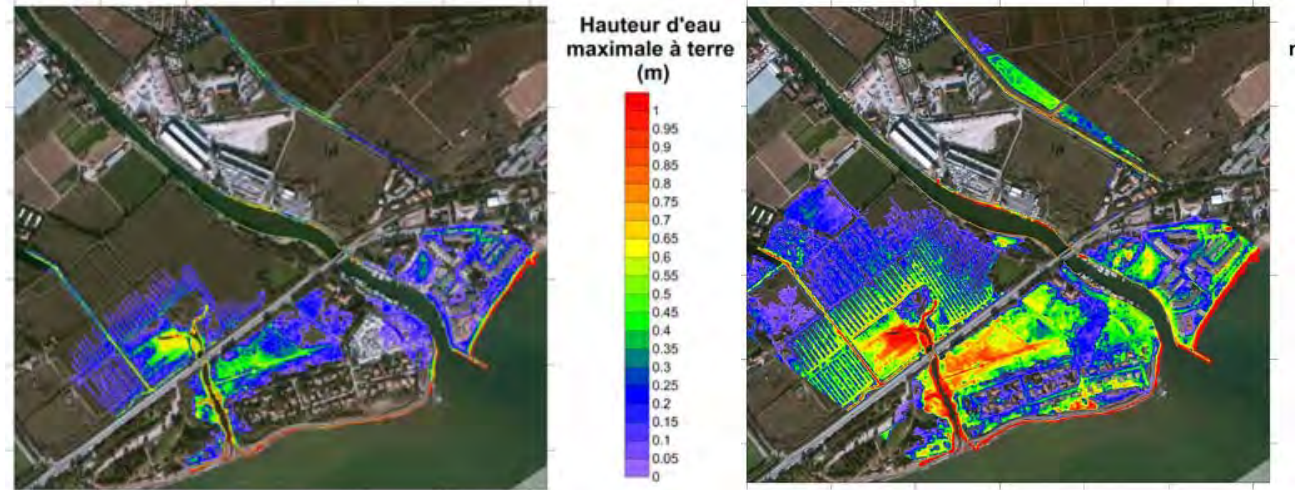

Figure 6. Hauteurs d'eau maximales simulées à terre aux Cabanes du Gapeau pour les scénarios trentennal échéance 203 (gauche) et centennal échéance 2100 (droite).

\section{Conclusions}

Ces résultats, outre les conclusions spécifiques au site d'étude, montrent que les outils numériques récents et la disponibilité de données à très haute résolution permettent désormais de simuler de manière réaliste la complexité des phénomènes impliqués dans les submersions marines, y compris dans le contexte de petits estuaires. La 
quantification des conséquences du changement climatique devient ainsi envisageable (moyennant toutefois certaines hypothèses, ici par exemple que les statistiques réalisées sur l'actuel reste valable à l'avenir), et permet de montrer que certains secteurs a priori actuellement relativement épargnés par cet aléa pourraient à l'avenir devenir significativement exposés.

\section{Références bibliographiques}

BOOIJ N., HAAGSMA I.J.G., HOLTHUIJSEN L.H., KIEFTENBURG AT.M.M, RIS R.C., VAN DER WESTHUYSEN A.J., ZIJLEMA M. (2004). Swan Cycle III version 40.41. User's Manual, 115 p.

HR WALLINGFORD, LANCASTER UNIVERSITY (2000). The Joint Probability of Waves and Water Levels: JOIN-SEA - A rigorous but practical new approach. Report SR537.

LE ROY S., PEDREROS R., ANDRE C., PARIS F., LECACHEUX S., MARCHE F., VINCHON C. (2015). Coastal flooding of urban areas by overtopping: dynamic modelling application to the Johanna storm (2008) in Gâvres (France). Natural Hazards and Earth System Sciences, Vol. 15, pp 2497-2510. http://dx.doi.org/10.5194/nhess-15-2497-2015

STEPANIAN A., LECACHEUX S., NICOLAE-LERMA A., PEDREROS R. (2014). Evaluation des Risques Naturels Littoraux sur le territoire du SCoT ProvenceMéditerranée - Caractérisation de l'aléa submersion marine. Rapport final. BRGM/RP-63949-FR, 119 p., 65 ill., 5 ann., 1 CD.

ZIJLEMA M., STELLING G., SMIT P. (2011). SWASH: An operational public domain code for simulating wave fields and rapidly varied flows in coastal waters. Coastal Engineering, Vol. 58(10), pp 992-1012. http://dx.doi.org/10.1016/j.coastaleng.2011.05.015 